\title{
Time-Resolved Chiral Vibrational Spectroscopy
}

\author{
Jan Helbing* and Mathias Bonmarin
}

\begin{abstract}
Advances in infrared laser technology and detection sensitivity have made time-resolved chiral vibrational spectroscopy experimentally feasible. Here we describe our recent efforts in detecting, for the first time, transient vibrational circular dichroism (VCD) signals with picosecond time resolution. The absorption changes of the cobalt (-)-sparteine complex $\mathrm{Co}(\mathrm{sp}) \mathrm{Cl}_{2}$ after visible excitation of a $d$ - $d$ excited state was probed in the $\mathrm{CH}$-stretch region by alternating left- and right-handed circular polarized mid IR laser pulses. VCD spectra can be sensitive reporters of peptide and protein secondary structure or the absolute configuration of chiral organic compounds in solution. Recent developments are presented, which may in the future allow us to access this information in the course of fast chemical reactions.
\end{abstract}

Keywords: Chirality · Molecular structure · Time-resolved spectroscopy · Vibrational circular dichroism

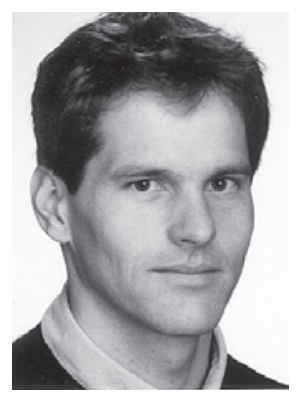

Jan Helbing was born in Germany and studied physics in Würzburg where, following a master's degree from Stony Brook, New York, he completed his diploma work with Prof. Gerber in 1996. For his $\mathrm{PhD}$ work he joined the group of Prof. Chergui at the University of Lausanne. Early in 2002 he moved to the group of Prof. Hamm at the University of Zürich as postdoc, developing transient $2 \mathrm{D}-\mathrm{IR}$ methods. Since November 2004 he is Oberassistent at the Institute of Physical Chemistry, investigating novel photoswitches for peptides and developing transient VCD spectroscopy.

${ }^{\star}$ Correspondence: Dr. J. Helbing

University of Zürich

Institute of Physical Chemistry

Winterthurerstrasse 190

$\mathrm{CH}-8057$ Zürich

Tel.: +41446354471

Fax: + 41446356838

E-mail: j.helbing@pci.uzh.ch

\section{Introduction}

Chiroptical probing of dynamic processes in molecules is potentially very attractive because of the enhanced structure sensitivity of techniques such as circular dichroism $^{[1]}$ or Raman optical activity ${ }^{[2]}$ with respect to conventional absorption or scattering experiments.

Early implementations of electronic time-resolved circular dichroism spectroscopy used flash photolysis ${ }^{[3]}$ temperaturejump ${ }^{[4]}$ or stopped flow techniques ${ }^{[5]}$ to study conformational kinetics of proteins on a millisecond timescale. Complementary to dichroism measurements (probing the difference in absorption of left- and righthanded circular polarized light) changes in optical rotation could also be detected. ${ }^{[6]}$ The use of pulsed lasers for photo triggering has made it possible to resolve transient changes in circular dichroism first with nanosecond ${ }^{[7]}$ and later picosecond ${ }^{[8]}$ time-resolution (see ref. [9] for a review of the initial development of the field). Only very recently sub-picosecond time resolution was achieved in the ultraviolet.[10] While kinetics on the timescale of hundreds of nanoseconds or slower can still be studied using flash lamps or continuous sources in combination with fast electronics, sub-nanosecond dynamics can only be resolved when the probe light itself is a short laser pulse. These typically have a bandwidth of only a few nanometres in the $\mathrm{UV}$, which limits the spectral window that can be probed in a single experiment.

In the mid-infrared, on the other hand, broad band femtosecond laser pulses cover multiple vibrational transitions in a spectral region of a few hundred wavenumbers and are easily tuneable from the fingerprint region up to the $\mathrm{C}-\mathrm{H}$ stretch vibrations. ${ }^{[11]}$ Transient vibrational spectroscopy has thus become a versatile tool for the investigation of reaction dynamics in solution, ${ }^{[12]}$ often aided by reliable quantum chemistry calculations in the electronic ground state. The combination of experiment and theory is even more powerful in the case of chiral vibrational spectroscopies like vibrational circular dichroism (VCD) or Raman optical activity (ROA), by which it is possible to determine the absolute configuration of organic compounds in solution, ${ }^{[13-16]}$ the conformation of polypeptides ${ }^{[17-20]}$ or the secondary structure content of proteins. ${ }^{[19]}$ Isotope labelling can be used to single out special regions of interest in larger molecules in order to obtain local structural information. ${ }^{[18]}$

However, unlike in the electronic case, time-resolved chiroptical measurements of vibrational transitions have not been attempted until very recently, and even static experiments can still be challenging. Indeed, since circular dichroism signals scale like the inverse of the probe wavelength they are much more difficult to detect for vibrational transitions in the mid-infrared than for electronic transitions in the UV, and the difference in absorption of left- and right-handed circular polarized light is typically only $10^{-3}-10^{-5}$ of the total absorption.

In this contribution we discuss a conceptually straightforward approach, by which we have recently been able to detect transient VCD changes for the first time. ${ }^{21]}$ Alternative methods which are currently being developed both in Zürich and in other groups for both vibrational and electronic transient circular dichroism spectroscopy are summarized. We do not 
discuss the expanding field of non-linear spectroscopic methods for the detection of chiral molecules, for which the reader is referred to recent review articles.[22,23]

\section{Vibrational Circular Dichroism and Molecular Structure}

Circular dichroism and optical rotation phenomena are described by considering the spatial variation $e^{i \vec{k} \cdot \vec{r}}$ of the electromagnetic field on the length scale of the molecule to lowest order. This leads to new terms in the field-matter Hamiltonian, which are proportional to $\vec{k} \cdot \vec{r} \approx 2 \pi r / \lambda$, i.e. the ratio of the extension of the charge distribution and the light wavelength, and can be written as magnetic field-magnetic dipole and electric field-electric quadrupole interactions:[24]

$$
\begin{aligned}
H_{\mathrm{int}} & =-e p \cdot \vec{A}_{0} e^{-i(\omega t-\vec{k} \cdot \vec{r})} \\
& \approx-e p \vec{A}_{0} e^{-i \omega t}(1+i \vec{k} \cdot \vec{r}) \\
& =-\vec{\mu} \cdot \vec{E}-\vec{m} \cdot \vec{B}-\sum_{\alpha, \beta} Q_{\alpha \beta} \nabla_{\alpha} E_{\beta}
\end{aligned}
$$

When linear polarized light interacts with an isotropic ensemble of molecules, the leading electric dipole term $\vec{\mu} \cdot \vec{E}$ describes the creation of a linear polarization (charge oscillation) and the emission of a field (the free induction decay) polarized in the same direction. In resonance, this field is out of phase with the incident one, leading to destructive interference, i.e. the absorption of light. Contributions of the quadrupole term cancel in isotropic samples, but those of the magnetic dipole term $\vec{m} \cdot \vec{B}$ do not when the molecules are chiral. In resonance with a transition (with both non-vanishing magnetic and electric transition dipole moments), it accounts for a free induction decay component with amplitude proportional to $\vec{\mu} \cdot \vec{m}$ that is polarized perpendicular to the incident field and phaseshifted by one quarter of an optical cycle. Linear polarized light thus turns into elliptically polarized light when passing a chiral sample. When the incident beam is circular polarized, its $x$-polarised component induces a $y$-polarised free induction decay which, due to the quarter-wave phase shift, interferes either destructively or constructively with the $y$-component of the incident field (and vice versa), leading to the different absorption cross sections for left- and righthanded circular polarizations.

Circular dichroism can thus be viewed as a result of charge motion induced perpendicular to the polarization of the incident electric field, which provides an intuitive understanding of the structure sensitivity of vibrational circular dichroism applied to peptides and proteins. Their prominent amide I normal modes can be well described as a linear combination of

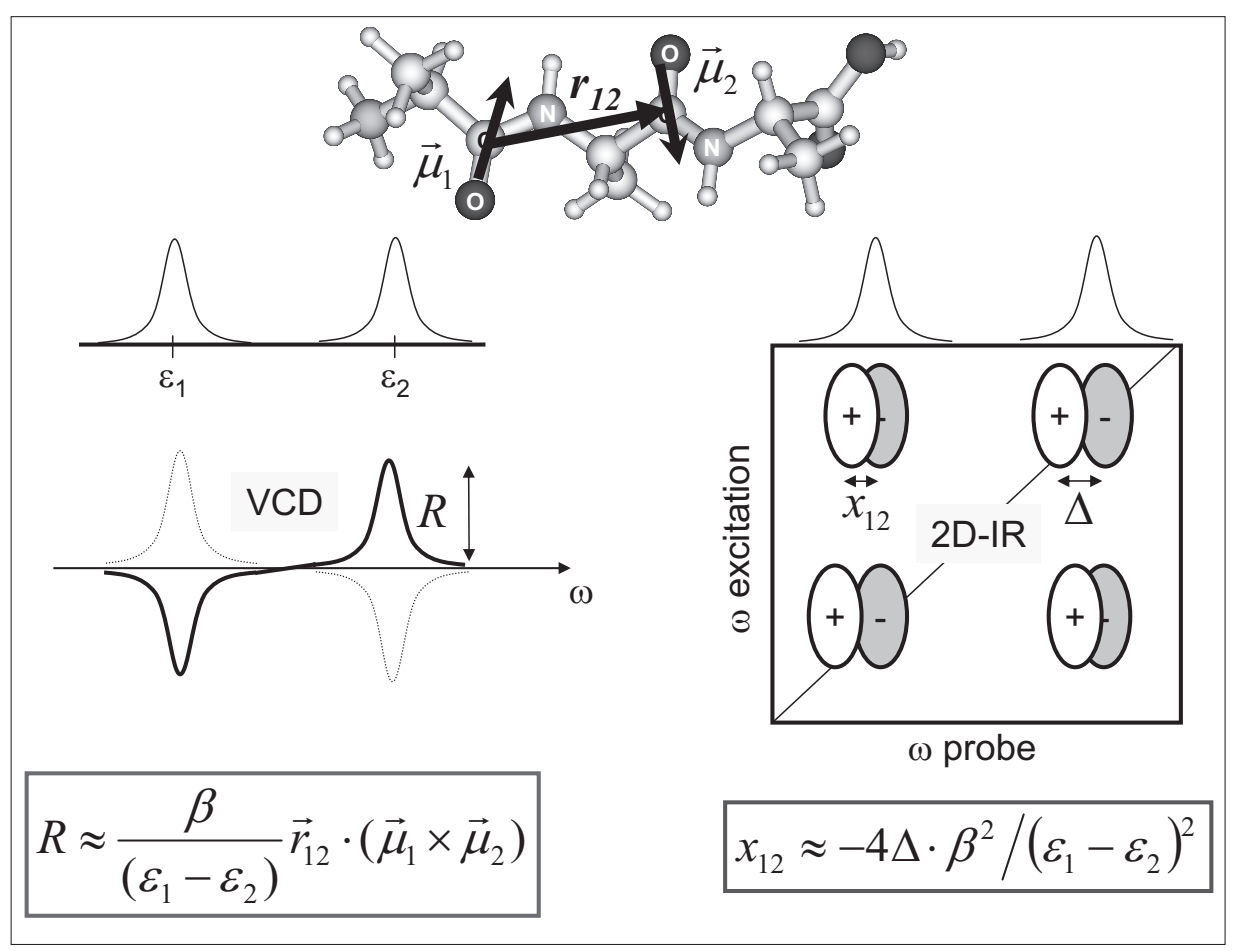

Fig. 1. Left: Schematic absorption and VCD spectrum of two coupled amide I oscillators with electric transition dipole moments $\vec{\mu}_{1}$ and $\vec{\mu}_{2}$ and energies $\varepsilon_{1}$ and $\varepsilon_{2}$, separated by a distance $r_{12}$. The pattern shown (negative VCD at lower frequency) is characteristic of polyproline II and 'random coil' conformations, whereas $\alpha$-helices exhibit the opposite pattern (dotted line). Similar information on angles and the coupling strength $\beta$ is contained in the cross peaks of a twodimensional IR spectrum, schematically shown at the right.

coupled, essentially localized $\mathrm{C}=\mathrm{O}$ stretch motions. ${ }^{[25]}$ When one $\mathrm{C}=\mathrm{O}$ group is driven by an external electric field, neighbouring carbonyls also oscillate to an extent proportional to the coupling constant $\beta,{ }^{[26]}$ and they contribute a perpendicular free induction decay component whose sign and magnitude depends on the relative orientation of the $\mathrm{C}=\mathrm{O}$ moieties (see Fig. 1).

It is interesting to note that circular dichroism, a linear spectroscopic technique, is sensitive to parameters that are otherwise only accessible by non-linear methods. In particular, two-dimensional infrared spectroscopy has recently been used to determine amide I coupling constants and the secondary structure propensity of small peptides. ${ }^{[27-30]}$ Both techniques therefore promise enhanced structural information when used to probe (photo-triggered) fast chemical reactions and conformational change in biomolecules. [31-34]

\section{Experimental Setup}

We obtain our mid-infrared light pulses by frequency mixing the signal and idler output of an optical parametric amplifier (OPA) that is pumped by $800 \mathrm{~nm}$ light from an amplified Ti:sapphire femtosecond laser system (Fig. 2). Visible (600 nm) pulses for electronic excitation in the pump-probe experiments are generated via similar four- wave mixing processes in another, noncollinear OPA pumped at $400 \mathrm{~nm}$.

The bandwidth of the mid-IR pulses is narrowed to the desired spectral resolution in a home-built Ebert-Fastie monochromator. The probe pulses subsequently pass a wire-grid polarizer (extinction ratio of approximately $10^{-4}$ ) followed by a photoelastic modulator (PEM). This is a $\mathrm{ZnSe}$ bar which is periodically compressed by piezoelements at its eigenfrequency of $50 \mathrm{kHz}$, to which the $1 \mathrm{kHz}$ laser system is synchronized. Each time an IR-pulse crosses the PEM it is at a turning points of its oscillation and acts as a quarter waveplate with alternating sign, producing either left- or right-handed circular polarized light.

In order to minimize artefacts which may arise from the polarization-sensitive transmission or reflection of mirrors and lenses, the probe beam focus on the sample $(100 \mu \mathrm{m})$ is imaged onto a nitrogen-cooled single-element MCT (mercury cadmium telluride) detector by a single lens.

The intensity of each laser pulse is recorded individually by time-gated integration and read into a computer with 16-bit resolution. For intensity normalization a reference beam is recorded separately (see Fig.2) and the VCD signal is given by:

$$
\Delta \alpha_{V C D}=\log _{10} \frac{I_{L}}{I_{R}} \frac{I_{r e f(R)}}{I_{r e f(L)}}
$$




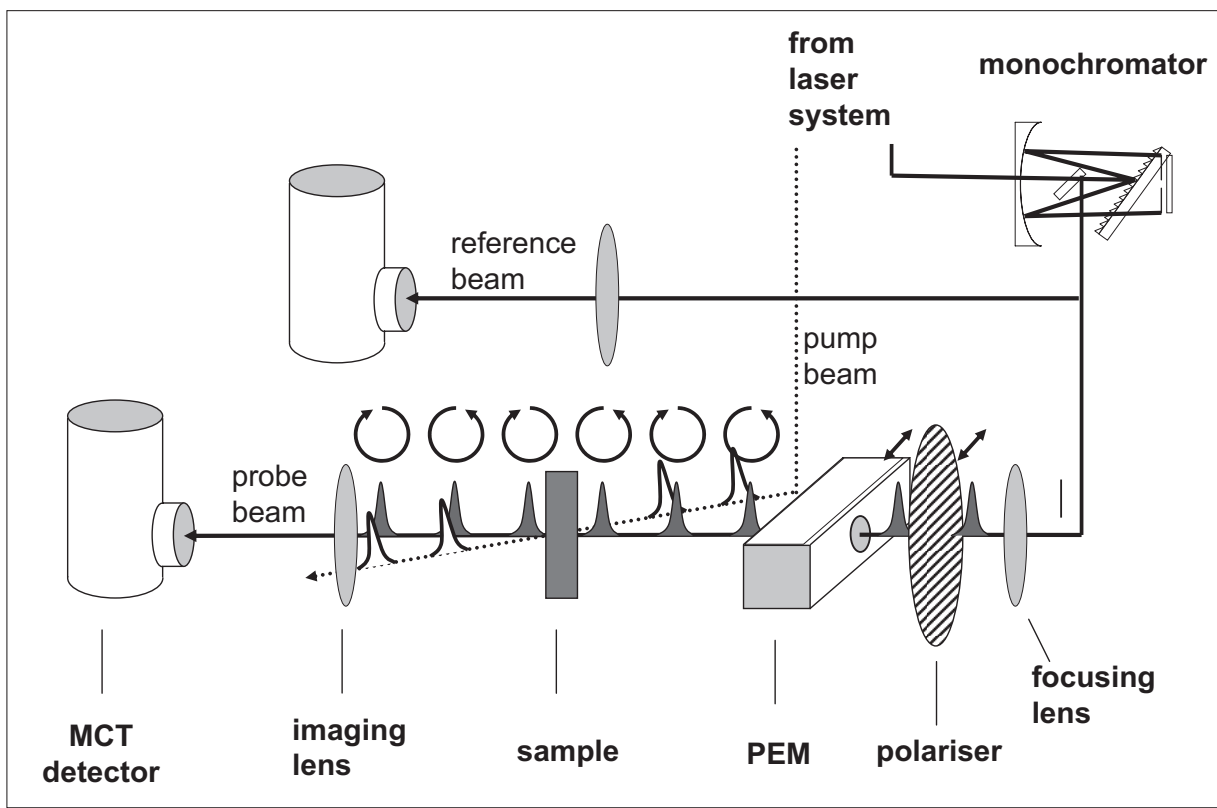

Fig. 2. Schematic view of the transient VCD setup in Zurich. The intensity of alternating left and right handed circular polarized light pulses is recorded with and without a preceding visible pump pulse. Polarization states of the probe pulses are indicated by arrows.

Typically a noise level of $20 \mu \mathrm{OD}$ is reached after averaging over 1000 laser pulses $(1 \mathrm{sec})$ at a given probe wavelength. Spectra are assembled by rotating the grating of the monochromator.

For the transient measurements the visible pump-beam used to trigger the chemical reaction is chopped at one quarter of the laser repetition rate. One pair of left- and right-handed probe pulses thus probes the VCD of the sample in the presence of a preceding visible pulse, while the subsequent pair measures the VCD of the unexcited sample, which is continuously exchanged in a flow cell[35] (see Fig. 2). Only the comparison of these quasi-simultaneously measured signals allows us to reach the sensitivity necessary to observe photoinduced VCD changes.

\section{Proof of Principle Experiment}

Like any new spectroscopic technique, transient VCD measurements are best demonstrated on a model system promising relatively strong signals. Open shell transition metal complexes with (-)-sparteine proved well-suited for this purpose. The exceptionally large VCD signals of $\mathrm{Co}(\mathrm{sp})$ $\mathrm{Cl}_{2}$ and $\mathrm{Ni}(\mathrm{sp}) \mathrm{Cl}_{2}$ was first noted for the $\mathrm{C}-\mathrm{H}$ stretch transitions, ${ }^{[36]}$ which are superimposed on a broad, unstructured electronic signal. This background is absent, and the VCD is an order of magnitude weaker for $\mathrm{Zn}(\mathrm{sp}) \mathrm{Cl}_{2}$ (Fig. 3). A similar VCD enhancement for open shell transition metal atoms can also be observed in the fingerprint region ${ }^{[37]}$ and has been reported for other complexes of cobalt. It can be attributed to low-energy, magnetic $600 \mathrm{~nm}$ is responsible for the deep blue colour of the $\mathrm{Co}(\mathrm{sp}) \mathrm{Cl}_{2}$ complex. When designing the experiment, we expected that excitation of this electronic transition would temporarily change the $d$-electron configuration, shift the low energy electronic resonances and thus alter the enhancement of the vibrational CD signal. However, within the time-resolution of the experiment $(5 \mathrm{ps}$, limited by the probe bandwidth) we found no clear signature of this $d$ - $d$ excited state, which probably decays on a sub-picosecond timescale.

Nevertheless, we could observe transient VCD changes at different probe frequencies in the $\mathrm{C}-\mathrm{H}$ stretch region (Fig. 4A). These step-function-like chiral transients indicate a weakening of the negative dichroism in the ground state before excitation, and they strongly differ from the simultaneously recorded conventional transient absorption signals (Fig. 4B). The latter reflect an instantaneous shift of the underlying vibrational band to a lower frequency (Fig. 4D), which subsequently relaxes with a time-constant of 20 picoseconds, typical for a strongly heated molecule and the subsequent dissipation of vibrational energy to the solvent. ${ }^{[39]}$ The transient absorption spectrum after 150 ps can be reproduced by independent FTIR measurements of the absorption changes caused by an increase in sample temperature (thick dotted line in Fig. 4D). From the scaling factor we deduce a pump-laser-induced heating of the excited sample volume by approximately

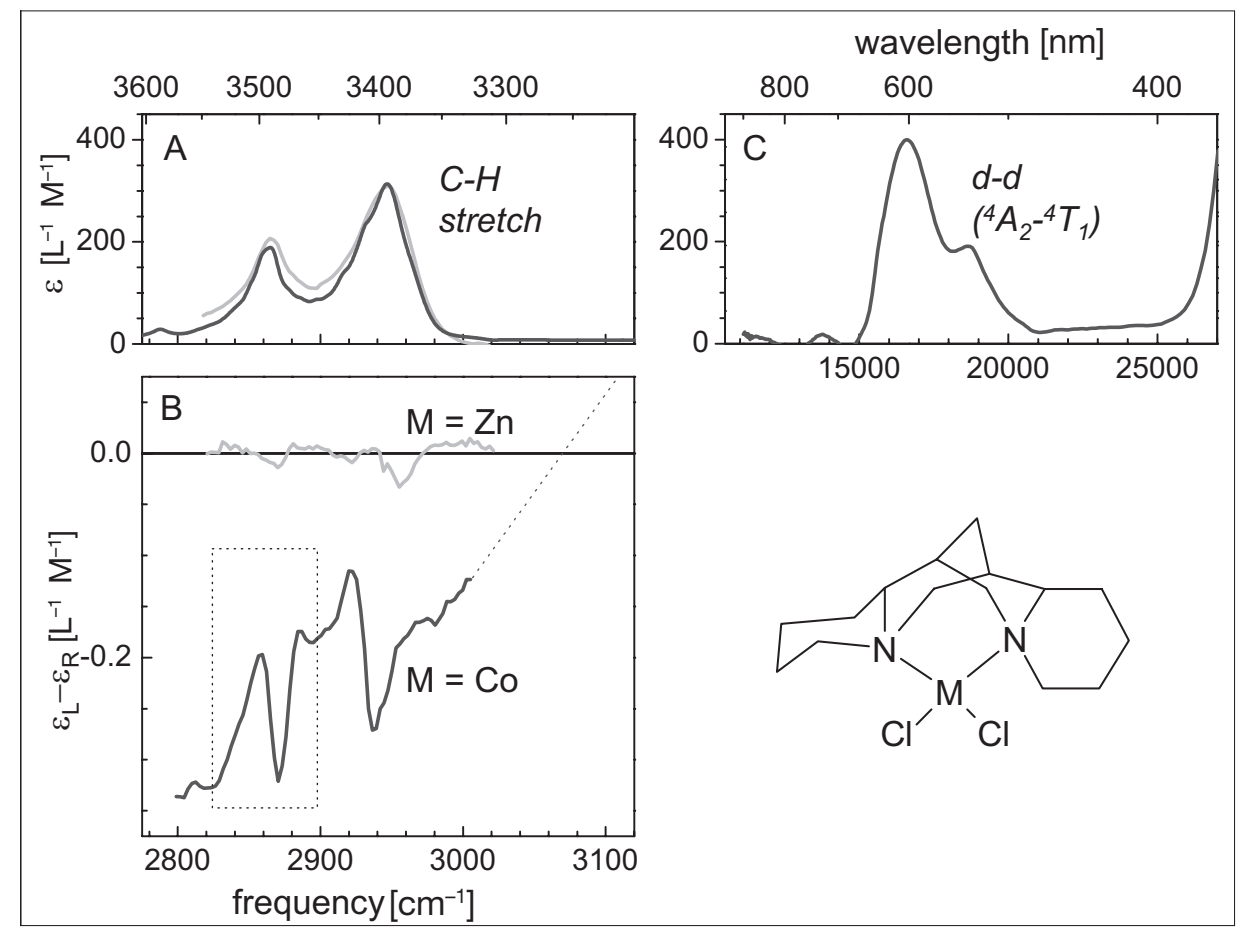

Fig. 3. A) Infrared absorption spectrum of $\mathrm{Co}(\mathrm{sp}) \mathrm{Cl}_{2}$ and $\mathrm{Zn}(\mathrm{sp}) \mathrm{Cl}_{2}$ in deuterated chloroform. B) VCD spectrum of the cobalt and zinc sparteine complexes. The 10 times stronger VCD of $\mathrm{Co}(\mathrm{sp}) \mathrm{Cl}_{2}$ is superimposed on a broad electronic signal (indicated by the dashed line outside the spectral window defined by the femtosecond probe pulses). The rectangle marks the spectral region probed in the transient measurements (see Fig 4). C) Visible absorption of $\mathrm{Co}(\mathrm{sp}) \mathrm{Cl}_{2}$ showing the band used for photo excitation. Optical densities ranged from $0.5-1.2$ OD in a 100 um flow cell. 


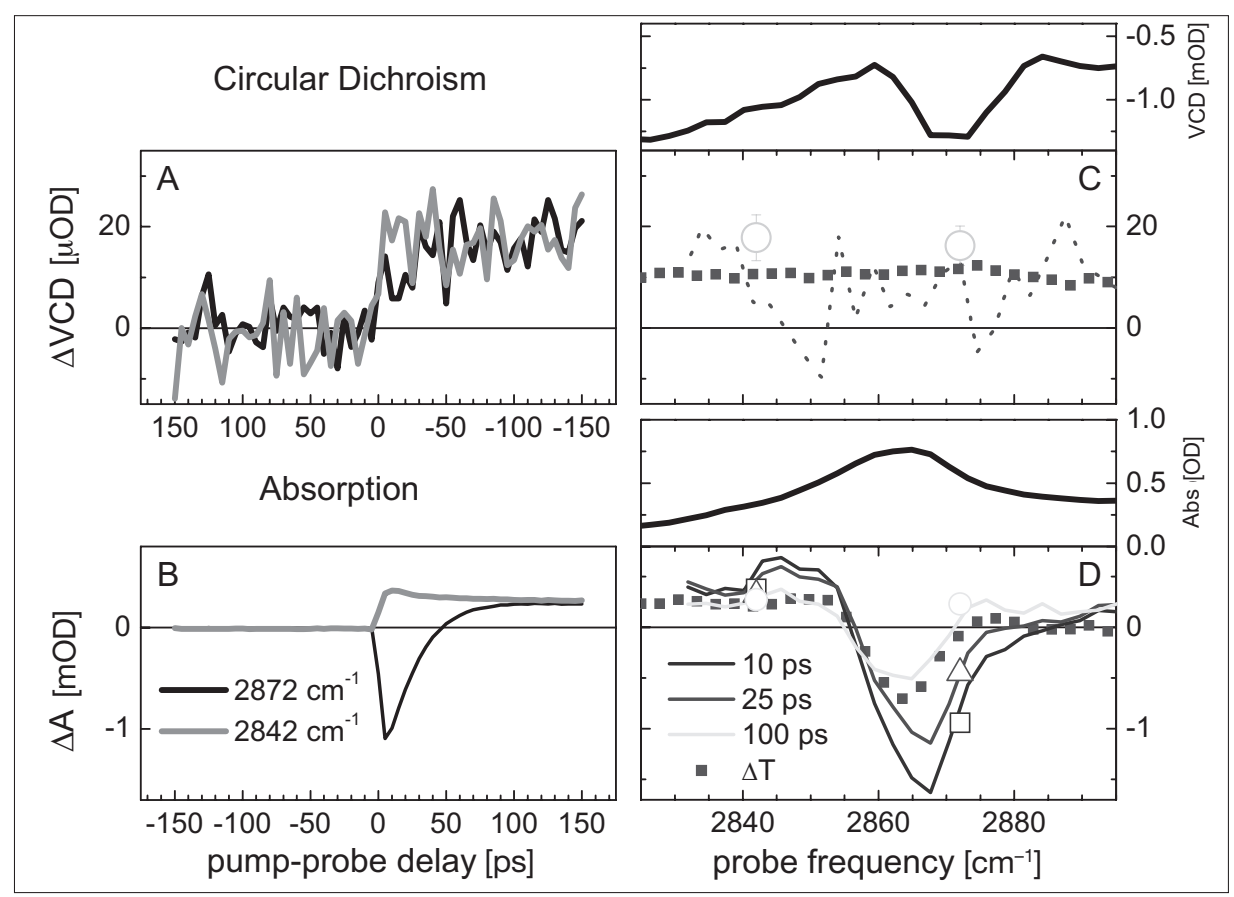

Fig. 4. A) Transient vibrational circular dichroism signal of $\mathrm{Co}(\mathrm{sp}) \mathrm{Cl}_{2}$ for excitation at $600 \mathrm{~nm}$ at probe frequencies of 2872 and $2842 \mathrm{~cm}^{-1}$. B) Simultaneously recorded transient absorption changes. C) Change in static circular dichroism due to a temperature rise of $0.7 \mathrm{~K}$ (thick dotted line). Open symbols show the transient VCD signals at a delay of 150 ps. D) Transient absorption spectra at different pump-probe delays. Open symbols are points from the transient scans shown in B. Thick dotted line: Changes in linear absorption caused by a $0.7 \mathrm{~K}$ temperature rise.

$0.7 \mathrm{~K}$. The equilibrium VCD of $\mathrm{Co}(\mathrm{sp})$ $\mathrm{Cl}_{2}$ is also temperature-dependent, growing weaker by $15 \mu \mathrm{OD} / \mathrm{K}$ near $2850 \mathrm{~cm}^{-1}$. As shown by the red dotted line in Fig $4 \mathrm{C}$ the VCD change corresponding to a temperature rise of $0.7 \mathrm{~K}$ agrees well with the magnitude of the chiral transients.

One hundred picoseconds after electronic excitation of only about $2 \%$ of the complexes in the probed sample volume the energy of the pump pulse has dissipated to the solvent and also those molecules, which were not directly excited, contribute to the observed absorption and dichroism change. In order to explain the essentially flat transient VCD signal we need to assume that the contribution of more and more molecules compensates for the cooling of the initially excited ones.

Finally, the thin dotted line in Fig. 4C shows a transient VCD 'spectrum' obtained by scanning the monochromator at a fixed pump-probe delay of $25 \mathrm{ps}$. After $1 \mathrm{~h}$ integration time the signal to noise is still close to unity and not sufficient to detect vibrational structure. Further technical improvements beyond our proof of principle experiment are therefore required needed, in particular for applications to biomolecular dynamics.

\section{Photo-triggered Conformational Transitions of Peptides}

The difference in molar extinction coefficient for left- and right-handed circu- lar polarized light of the cobalt sparteine complex is $\Delta \varepsilon \approx 0.5 \mathrm{~L}^{-1} \mathrm{M}^{-1}$, which allowed us to carry out transient measurements on samples with a static VCD signal of $0.5-1$ mOD. For polypeptides and proteins, on the other hand, $\Delta \varepsilon$ is typically ten times smaller (per mole amino acid). In comparison, the electronic CD signal of an $\alpha$-helix at $222 \mathrm{~nm}$ corresponds to $\Delta \varepsilon \approx 10 \mathrm{~L}^{-1} \mathrm{M}^{-1}$ per residue. However, helix-coil transitions lead to maximal changes in the VCD spectrum, because the sign of the characteristic amide I couplet is reversed (see Fig. 1). For example, Keiderling and co-workers have ${ }^{13} \mathrm{C}=\mathrm{O}$ isotope-labelled sections of an $\alpha$-helical peptide, allowing them to investigate the local secondary structure content as a function of temperature with VCD. [18] Wolley and co-workers have introduced azobenzene-based cross-linkers between cysteine side chains of similar peptides, ${ }^{[40]}$ which makes it possible to switch on and off helical structure by light. ${ }^{[41]}$ Photo-induced helix-unfolding could thus already be studied by nanosecond transient optical rotation (ORD) spectroscopy in the UVvisible spectral range, ${ }^{[42]}$ and much more detailed information can be expected from transient vibrational circular dichroism measurements. Azobenzene-based photoswitches have also been incorporated in a variety of other peptide structures, ${ }^{[43,44]}$ including $\beta$-sheets. ${ }^{[45,46]}$ Because the $\mathrm{C}=\mathrm{O}$ chromophores in $\beta$-sheets are oriented close to (anti-)parallel, vibrational circular dichroism is, however, comparatively weak, ${ }^{[23]}$ and other probes like 2D-IR may be more sensitive in this case. ${ }^{[47]} \mathrm{An}$ interesting alternative method for lighttriggering fast conformational change is the incorporation of a thiopeptide bond by substitution of one backbone carbonyl Catom by sulphur. The thiopeptide unit can then be excited selectively by a UV-pulse, which leads to the isomerization of the peptide bond from trans to $c$ is within a few hundred picoseconds. ${ }^{[48,49]}$ It has recently been shown that this isomerization near the $\mathrm{N}$-terminus of the $\mathrm{S}$ peptide in the protein complex ribonuclease $\mathrm{S}$ induces conformational changes sufficient to switch off enzymatic activity. ${ }^{[50]}$

In many of these examples photo-excitation of a significant fraction of molecules is possible, resulting in large conformational changes and transient VCD signal levels can be similar to those observed in the $\mathrm{Co}(\mathrm{sp}) \mathrm{Cl}_{2}$ model compound. In addition, the development of alternative detection schemes is about to further improve signal to noise levels in transient circular dichroism measurements.

\section{Technical Developments}

Our current approach, based on the differential absorption of left- and right-handed circular polarized light pulses, constitutes the most intuitive way of measuring circular dichroism. Compared to state-ofthe-art transient absorption experiments, where the probe pulses are dispersed after the sample and full spectra are recorded simultaneously by an array detector, scanning a narrow-band probe pulse may nevertheless look like a poor solution. However, we carefully chose this arrangement, because polarization-sensitive optics (like mirrors and gratings) affect left- and righthanded polarized light very differently, leading to signal distortions and offsets in static VCD measurements. They also aggravate pump-laser induced linear dichroism or birefringence artefacts, which can seriously perturb transient CD scans. [51] The latter are of concern even with our method and are negligible only because of a very high degree of symmetry of left- and right-handed circular probe light polarizations. ${ }^{21,52]}$

On the other hand, when these artefacts are properly controlled, important advantages may be gained from placing a crossed linear polarizer behind the sample. ${ }^{[53]}$ Intense linear polarized laser light can then be used to induce a strong free induction decay without saturating the detector. The perpendicular polarized chiral FID component is selectively transmitted by the second polarizer (see Section 2 and Fig. 5) and detected together with only a small portion of the incident light in order 


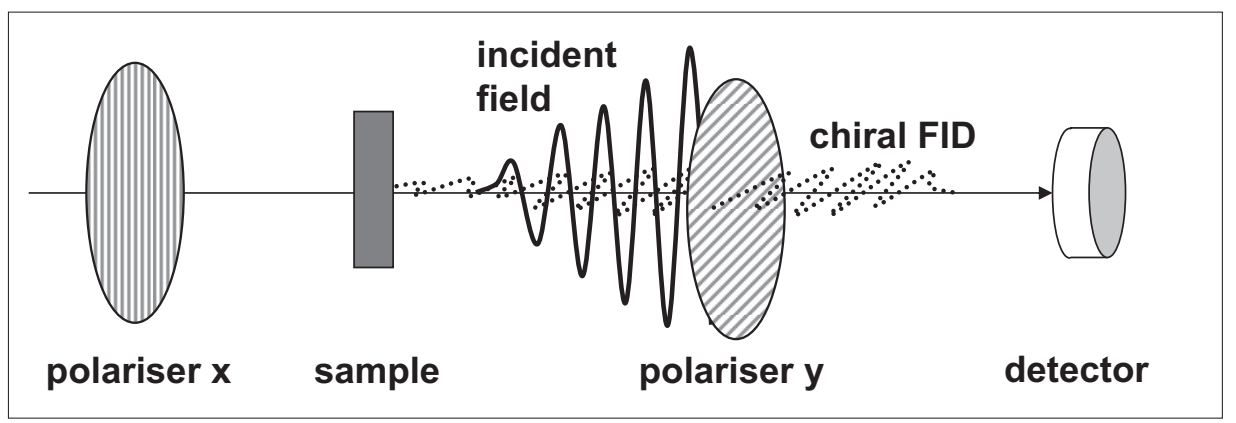

Fig. 5. Principle of VCD measurements with linearly polarised light. Most of the intense incident field (solid line) is blocked by a second polarizer to avoid detector saturation. The perpendicularly polarized free induction decay component emitted by chiral samples (dotted line) reaches the detector and can be amplified by different interferometric techniques (see text).

to distinguish resonant circular dichroism from off-resonant optical rotation. In this way the chiral contribution becomes a larger fraction of the detected signal and it is no longer measured as a tiny difference between two very large intensities. Crossed polarizer schemes are already used in nanosecond electronic $\mathrm{CD}^{[7]}$ and ORD $^{[54]}$ spectroscopy by Kliger and coworkers, and an interesting variant was recently introduced by Niezborala and Hache. ${ }^{[55]}$ In their setup a linearly polarized probe beam passes the sample, followed by a waveplate with small, variable birefringence (Babinet-Soleil compensator) and a crossed polarizer before being detected. The transmitted light intensity as a function of Babinet-Soleil retardation is a parabola. For chiral samples, this parabola is shifted horizontally from zero retardation although usually too little for a reliable measurement of static $C D$ signals. In transient measurements, however, the parabola shift is proportional to $\Delta \mathrm{CD} / \Delta \mathrm{A}$, where $\Delta \mathrm{A}$ and $\Delta \mathrm{CD}$ are the pump-induced absorbance and $C D$ changes. Thus, if accompanied by only small transient absorbance changes, the chiral signal is greatly enhanced and more easily measurable. The transfer of this concept to transient vibrational circular dichroism measurements in combination with broad band detection is currently pursued in our laboratory.

A related scheme for chiral mid-infrared spectroscopy was recently proposed by Cho and co-workers. ${ }^{[56]}$ Here the incident linearly polarized femtosecond laser beam is completely blocked by the crossed polarizer behind the sample. Only the purely chiral, perpendicular polarized free induction decay component is transmitted and detected interferometrically after superposition with a delayed replica of the incident beam that is guided around the sample. Both VCD and, for the first time, vibrational ORD spectra could be measured by this Fourier-type spectroscopy with unprecedented signal to noise. ${ }^{[57]}$
We are confident that these concepts will soon lead to more reliable transient VCD spectrometers, providing a new structure-sensitive probe for the investigation of very fast kinetics in chemistry and biology.

\section{Summary and Conclusions}

In this contribution we have focused on technical developments for the detection of transient vibrational circular dichroism signals and presented a recent proof of principle experiment. Possible future application to light-triggered conformational transitions of polypeptides and proteins were outlined. Main advantages of probing vibrational rather than electronic transitions in time-resolved CD experiments are universal applicability (every molecule has a vibrational spectrum), site-specific information from local modes and the precision of quantum chemistry calculations in the electronic ground state. The very small signal levels are still a major drawback, but new detection schemes promise significant improvement.

Combined with femtosecond laser sources, vibrational circular dichroism is not the only chiral spectroscopy with potentially high time resolution. Raman signals have already been used to resolve sub-picosecond dynamics and these methods could in principle be extended to chiral transient Raman optical activity measurements. Sum-frequency generation is another very sensitive and intrinsically ultrafast technique, which allows to selectively record vibrational spectra of chiral molecules. ${ }^{[58,59]}$ In addition, multidimensional spectroscopic probes of chirality have been proposed. [60,61] As more and more spectroscopists realize the potential of chiral measurements, and ultrafast lasers are becoming widely available, many interesting developments and results are to be expected in the near future.

\section{Acknowledgements}

We gratefully acknowledge support for our research from the Swiss National Science Foundation (200021-111902 and 200020119814) and the Alfred Werner Legat of the University of Zürich.

Received: February 2, 2009

[1] K. Nakanishi, N. Berova, R. W. Woody, 'Circular Dichroism Principles and Applications', $\mathrm{VCH}$ Publishers, Inc., New York, 1994.

[2] L. D. Barron, L. Hecht, I. M. McColl, E. W. Blanch, Mol. Phys. 2004, 102, 731.

[3] F. A. Ferrone, J. J. Hopfield, S. E. Schnatterly, Rev. Sci. Inst. 1974, 45, 1392.

[4] P. Bayley, S. Martin, M. Anson, Biochem. Biophys. Res. Comm. 1975, 66, 303.

[5] P. M. Bayley, M. Anson, Biopolymers 1974, 13 , 401.

[6] D. M. Goodall, M. T. Cross, Rev. Sci. Inst. 1975, 46, 391.

[7] J. W. Lewis, R. F. Tilton, C. M. Einterz, S. J. Milder, I. D. Kuntz, D. S. Kliger, J. Phys. Chem. 1985, 89, 289.

[8] X. Xie, J. D. Simon, J. Phys. Chem. 1990, 94 8014

[9] J. W. Lewis, R. A. Goldbeck, D. S. Kliger, X. Xie, R. C. Dunn, J. D. Simon, J. Phys. Chem. 1992, 96, 5243

[10] T. Dartigalongue, C. Niezborala, F. Hache, Phys. Chem. Chem. Phys. 2007, 9, 1611.

[11] P. Hamm, R. A. Kaindl, J. Stenger, Optics Letters 2000, 25, 1798

[12] E. T. J. Nibbering, H. Fidder, E. Pines, Annu. Rev. Phys. Chem. 2005, 56, 337

[13] J. Haesler, I. Schindelholz, E. Riguet, C. G. Bochet, W. Hug, Nature 2007, 446, 526.

[14] J. Haesler, W. Hug, Chimia 2008, 62, 482.

[15] P. J. Stephens, F. J. Devlin, Chirality 2000, 12, 172.

[16] T. B. Freedman, X. Cao, R. K. Dukor, L. A. Nafie, Chirality 2003, 15, 743.

[17] R. Schweitzer-Stenner, F. Eker, K. Griebenow, X. Cao, L. A. Nafie, J. Am. Chem. Soc. 2004, $126,2768$.

[18] R. Silva, J. Kubelka, P. Bour, S. M. Decatur, T. A. Keiderling, Proc. Natl. Acad. Sci. U.S.A. 2000, 97, 8318.

[19] T. A. Keiderling, J. Kubelka, J. Hilario in 'Vibrational Circular Dichroism of Biopolymers. Summary of Methods and Applications', Vol. Eds. M. Brainman, V. Gregoriou, Marcel Dekker, New York, 2005, p. 253.

[20] I. H. McDoll, W. W. Blanch, A. C. Gill, A. G. O. Rhie, M. A. Ritchie, L. Hecht, K. Nielsen, L. D. Barron, J. Am. Chem. Soc. 2003, 125, 10019.

[21] M. Bonmarin, J. Helbing, Opt. Lett. 2008, 33, 2086.

[22] P. Fischer, F. Hache, Chirality 2005, 421.

[23] T. A. Keiderling, Curr. Opin. Chem. Biol. 2002, $6,682$.

[24] M. Cho, G. R. Fleming, S. Mukamel, J. Chem. Phys. 1993, 98, 5314.

[25] H. Torii, M. Tasumi, J. Chem. Phys. 1992, 96, 3379.

[26] G. Holzwarth, I. Chabay, J. Chem. Phys. 1972, 57, 1632 .

[27] S. Woutersen, P. Hamm, J. Phys. Chem. 2000, 104, 11316.

[28] S. Woutersen, R. Pfister, P. Hamm, Y. Mu, D. S. Kosov, G. Stock, J. Chem. Phys. 2002, 117, 6833.

[29] J. Wang, J. Chen, R. M. Hochstrasser, J. Phys. Chem. B 2006, 110, 7545 .

[30] M. T. Zanni, R. M. Hochstrasser, Curr. Opin. Struct. Biol. 2001, 11, 516.

[31] J. Bredenbeck, J. Helbing, C. Renner, R. Behrendt, L. Moroder, J. Wachtveitl, P. Hamm, J. Phys. Chem. B 2003, 107, 8654. 
[32] C. Kolano, J. Helbing, M. Kozinski, W. Sander, P. Hamm, Nature 2006, 444, 469.

[33] P. Hamm, J. Helbing, J. Bredenbeck, Annu. Rev. Phys. Chem. 2008, 59, 291.

[34] H. S. Chung, Z. Ganim, K. C. Jones, A. Tokmakoff, Proc. Natl. Acad. Sci. U.S.A. 2007, 104, 14237.

[35] J. Bredenbeck, P. Hamm, Rev. Sci. Inst. 2003, 74,3188 .

[36] C. J. Barnett, A. F. Drake, R. Kuroda, S. F. Mason, S. Savage, Chem. Phys. Lett. 1980, 70, 8.

[37] Y. He, X. Cao, L. A. Nafie, T. B. Freedman, J. Am. Chem. Soc. 2001, 123, 11320.

[38] L. A. Nafie, J. Phys. Chem. A 2004, 108, 7222.

[39] P. Hamm, S. M. Ohline, W. Zinth, J. Chem. Phys. 1997, 106, 519.

[40] J. R. Kumita, O. S. Smart, G. A. Woolley, Proc. Natl. Acad. Sci. U.S.A. 2000, 97, 3803.

[41] J. Bredenbeck, J. Helbing, J. R. Kumita, G. A Woolley, P. Hamm, Proc. Natl. Acad. Sci. U.S.A. 2005, 102, 2379.

[42] E. Chen, J. R. Kumita, G. A. Woolley, D. S Kliger, J. Am. Chem. Soc. 2003, 125, 12443.

[43] R. Behrendt, C. Renner, M. Schenk, F. Wang, J. Wachtveitl, D. Oesterhelt, L. Moroder, Angew. Chem., Int. Ed. 1999, 38, 2771.

[44] G. Mayer, A. Heckel, Angew. Chem., Int. Ed. 2006, 45, 4900 .

[45] A. Aemissegger, V. Krautler, W. F. van Gunsteren, D. Hilvert, J. Am. Chem. Soc. 2005, 127, 2929

[46] T. E. Schrader, W. J. Schreier, T. Cordes, F. O. Koller, G. Babitzki, R. Denschlag, C. Renner, M. Loweneck, S.-L. Dong, L. Moroder, P.
Tavan, W. Zinth, Proc. Natl. Acad. Sci. U.S.A. 2007, 104, 15729.

[47] A. W. Smith, A. Tokmakoff, J. Chem. Phys. 2007, 126, 045109 .

[48] J. Helbing, H. Bregy, J. Bredenbeck, R. Pfister, R. Huber, P. Hamm, J. Wachtveitl, L. D. Vico, M. Olivucci, J. Am. Chem. Soc. 2004, 126, 8823.

[49] V. Cervetto, R. Pfister, J. Helbing, J. Phys. Chem. B 2008, 112, 3540.

[50] D. Wildemann, C. Schiene-Fischer, T. Aumüller, A. Bachmann, T. Kiefhaber, C. Lücke, G. Fischer, J. Am. Chem. Soc. 2007, 129, 4910.

[51] X. Xie, J. D. Simon, J. Opt. Soc. Am. B 1990, 7, 1673.

[52] M. Bonmarin, J. Helbing, 2009, in preparation.

[53] R. A. Goldbeck, D. B. Kim-Shapiro, D. S. Kliger, Annu. Rev. Phys. Chem. 1997, 48, 453.

[54] E. Chen, Y. Wen, J. W. Lewis, R. A. Goldbeck, D. S. Kliger, Rev. Sci. Inst. 2005, 76, 083120.

[55] C. Niezborala, F. Hache, J. Opt. Soc. Am. B 2006, 23, 2418.

[56] H. Rhee, J.-H. Ha, S.-J. Jeon, M. Cho, J. Chem. Phys. 2008, 129, 094507.

[57] M. Cho, oral presentation, CMDS Conference, Kyoto, Aug. 2008.

[58] N. Ji, Y.-R. Shen, Chirality 2006, 18, 146.

[59] N. Ji, Y. R. Shen, J. Am. Chem. Soc. 2004, 126, 15008.

[60] D. Abramavicius, S. Mukamel, J. Chem. Phys. 2005, 122.

[61] J. H. Choi, M. Cho, J. Phys. Chem. A 2007, 111, 5176. 